\title{
Pressure neuropathy in the hind foot of the guinea-pig
}

\author{
PAMELA M. FULLERTON ${ }^{1}$ AND R. W. GILLIATT \\ From the Institute of Neurology, Queen Square, London
}

Patients who regularly exert pressure with the palm of the hand may develop a lesion of the deep branch of the ulnar nerve. The hypothenar muscles are usually spared, weakness and wasting being confined to the interossei and adductor pollicis (Russell and Whitty, 1947). In such cases conduction velocity is markedly reduced in the terminal parts of the motor nerve fibres (Simpson, 1956; Ebeling, Gilliatt, and Thomas, 1960) but the underlying pathological changes in the nerves have not been studied in man as no material has been available. The purpose of the present paper is to report a localized peripheral nerve lesion in the guinea-pig which shows some similarities with the human condition described above.

The animal lesion was discovered in the course of some experiments in which guinea-pigs were kept in small cages on wire-mesh flooring for periods of up to a year. Abnormalities of conduction were observed in nerves supplying the small muscles of the hind foot, suggesting that the wire-mesh floors of the cages might have produced local damage to the plantar nerves (Fullerton and Gilliatt, 1965). This phenomenon has now been further investigated.

\section{METHODS}

Healthy male and female guinea-pigs aged 3-24 months were studied. The animals may be divided into four groups:

A Young guinea-pigs, aged 3-6 months, which had been reared and kept in large pens with solid floors.

B Old guinea-pigs, aged 18-24 months, which had been reared and kept in similar pens.

C Guinea-pigs which had been reared in large pens with solid floors and transferred, at ages varying from 2 to 16 weeks, to small cages with wire-mesh floors, in which they were kept for further periods of eight to 12 months. The cages measured $25 \times 40 \mathrm{~cm}$. The mesh flooring consisted of $1 \mathrm{~mm}$. gauge wire arranged in $1 \mathrm{~cm}$. squares.

D Guinea-pigs which were similar to those in group $\mathbf{C}$ except that they were transferred from large pens to small cages $(25 \times 40 \mathrm{~cm}$.) with solid floors covered by a deep layer of sawdust.

'Part-time member of the Scientific Staff, M.R.C. Toxicology Unit, Carshalton, Surrey.
All animals were fed a standard S.G. 1 pellet diet (Short and Gammage, 1959) supplemented by fresh cabbage.

Conduction velocity in the motor fibres to the small muscles of the hind foot was estimated in anaesthetized animals in a manner similar to that originally described by Kaeser and Lambert (1962). Details of the technique are given by Fullerton (1966).

Nerves for histology were carefully removed with the aid of a dissecting microscope and were then slightly stretched on card frames and fixed in Flemming's solution or $10 \%$ formol-saline. Nerves fixed in Flemming's solution were embedded in paraffin; transverse sections were cut at $5 \mu$, and sections stained by the modified Weigert method to demonstrate myelin sheaths (Gutmann and Sanders, 1943). Some sections were counterstained by the van Gieson method. Nerves fixed in formol-saline were examined in two ways. In most cases the nerves were stained with $1 \%$ osmium tetroxide and teased apart in glycerin using the technique described by Thomas (1955); single fibres were prepared under a dissecting microscope at a magnification of $\times 100$ and abnormal fibres preserved by mounting in Canada balsam for later examination at higher magnification. In a few cases nerves were embedded in paraffin and longitudinal sections were cut at $5 \mu$; these were stained by Glees and Marsland's modification of Bielschowsky's method (Culling, 1963) and counterstained with luxol fast blue.

\section{ELECTROPHYSIOLOGICAL RESULTS}

When the motor fibres supplying the small muscles of the hind foot were stimulated in the thigh and just above the ankle, and action potentials recorded from the interosseous muscles through belly-tendon electrodes, conduction velocity in the fastest motor fibres varied from 40 to $64 \mathrm{~m}$./sec. in different animals. The range of maximal velocity was similar in groups A, B, and C (Fig. 1, left). In young adult animals which had been reared and kept on solid floors (group A) distal latency varied from 1.4 to 2.2 msec. (Fig. 1, right), whereas in some of the older animals from group B higher values (up to $2.6 \mathrm{msec}$.) were obtained. In the guinea-pigs kept on wire-mesh floors (group C) a few animals showed longer distal latencies than either of the 

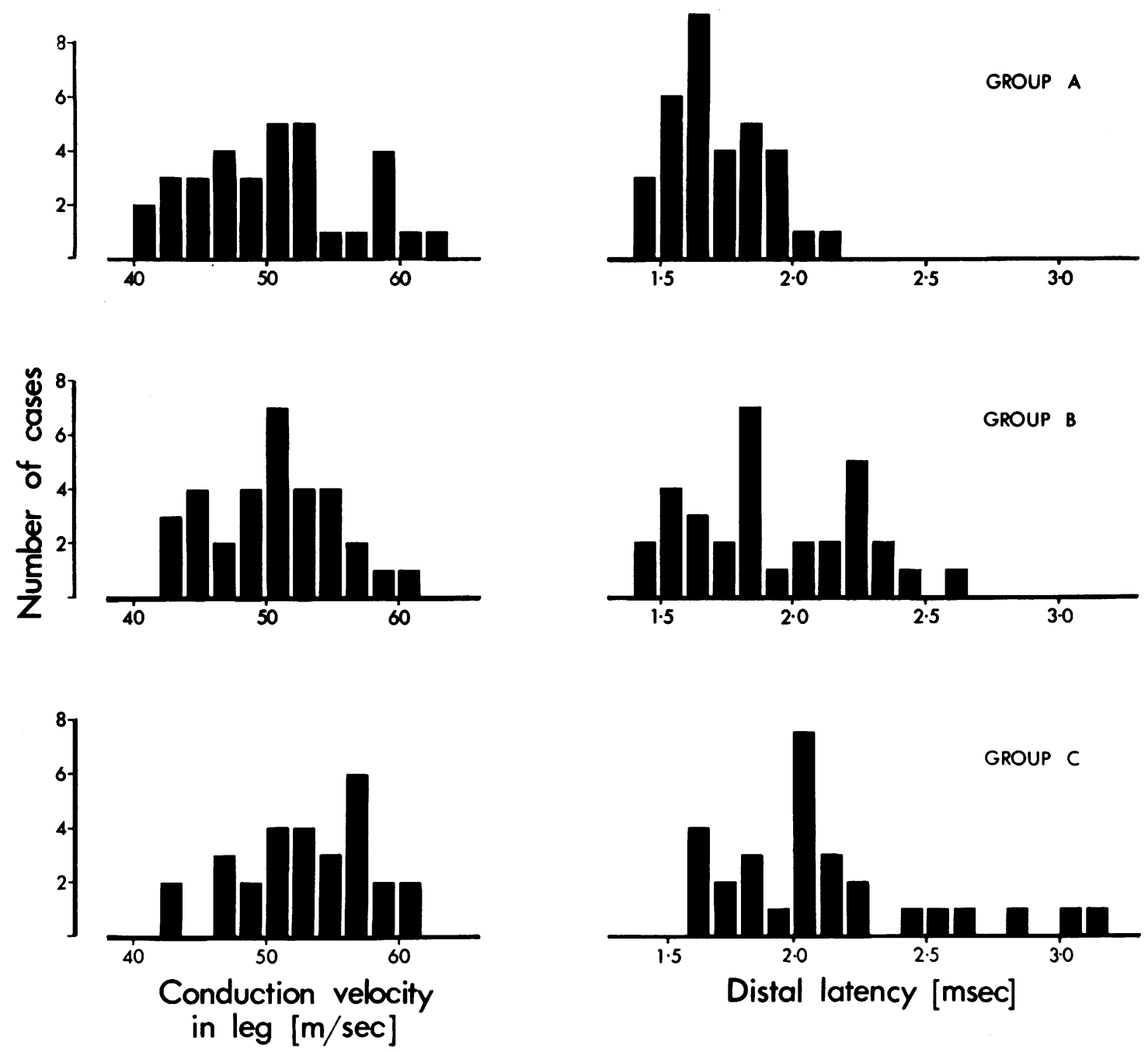

FIG. 1. Histograms to show conduction velocity in the leg and distal latency in different groups of guinea-pigs.

other two groups, values up to $3.2 \mathrm{msec}$. being obtained.

From these results it appears that while the use of wire-mesh flooring has no effect on maximal velocity in the leg itself, it may sometimes result in a slight increase in distal latency. However, a much more striking abnormality in animals on wire-mesh flooring was dispersion and prolongation of the muscle response to nerve stimulation. This is illustrated by Figure 2 which shows an experiment on a group $C$ animal in which muscle action potentials were also recorded from extensor digitorum brevis, the anterior tibial nerve being stimulated on the dorsum of the ankle. The simple biphasic potential obtained from extensor digitorum brevis may be contrasted with the long-duration polyphasic response recorded from the sole when the posterior tibial nerve was stimulated. There could be no question of this prolonged response being due to spinal reflex activity, as it persisted when the nerve was divided proximal to the stimulating cathode.

More information was obtained by the use of a double-core needle electrode (Disa no. 13 K80). When this was inserted into one of the interosseous muscles, single motor unit potentials could be identified by their characteristic waveform. In the example shown in Figure 3, weak shocks to the 


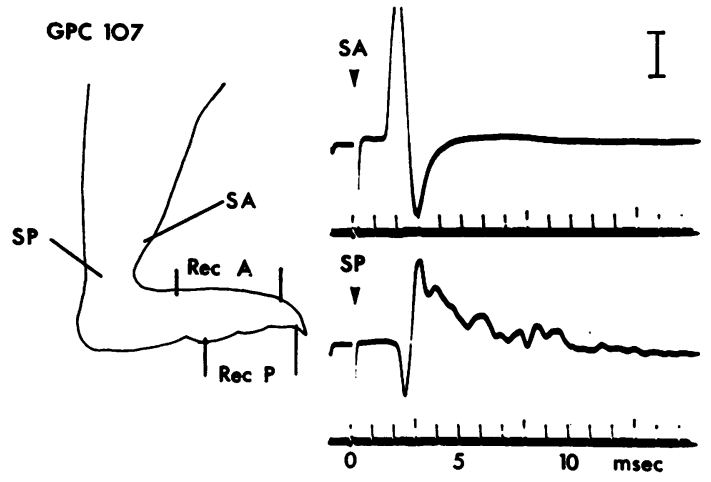

FIG. 2. GPC 107 (group C). Upper trace: muscle action potentials recorded from extensor digitorum brevis following stimulation of anterior tibial nerve at ankle $(S A)$. Lower trace: potentials recorded from interossei following stimulation of posterior tibial nerve at ankle $(S P)$. Recordings through belly-tendon electrodes. Calibration $-1 \mathrm{mV}$.

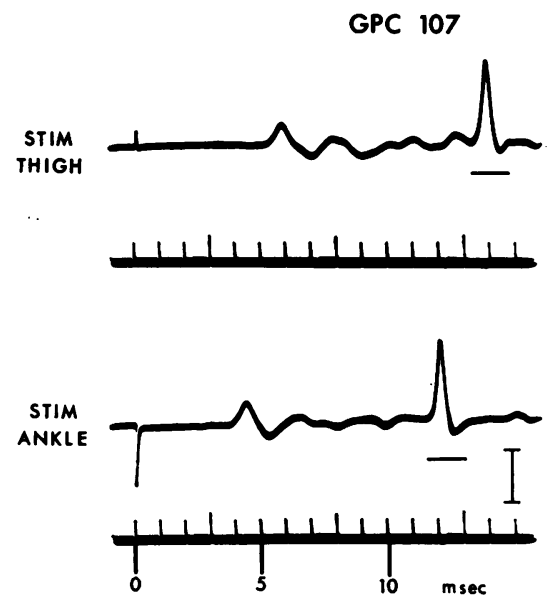

FIG. 3. A single motor unit action potential (underlined) from GPC 107 to show prolonged distal latency. A doublecore needle electrode was used for recording. Calibration $-1 \mathrm{mV}$.

nerve produced some low-voltage fluctuations of the trace due to distant muscle activity and in addition a single spike potential with a latency of $11.6 \mathrm{msec}$. with stimulation at the ankle and 13.3 msec. with stimulation in the thigh. This potential showed an all-or-none response to increasing stimulus intensity and may therefore be presumed to be due to the activity of a single motor unit close to the tip of the recording needle. Its greatly prolonged latency with ankle stimulation indicates a conduction delay in the foot rather than in the leg itself.
In four animals with dispersed muscle action potentials, the motor fibres to the small muscles of the foot were stimulated not only in the thigh and at the ankle but also on the medial side of the foot. At each level the cathode was a needle electrode placed close to the nerve trunk so that weak shocks could be used and current spread minimized. The interosseous muscles were explored with a doublecore needle electrode until both early and late motor-unit responses were obtained, these responses remaining constant with nerve stimulation at all three levels. The experimental arrangement and a typical result are shown in Figure 4. In this animal

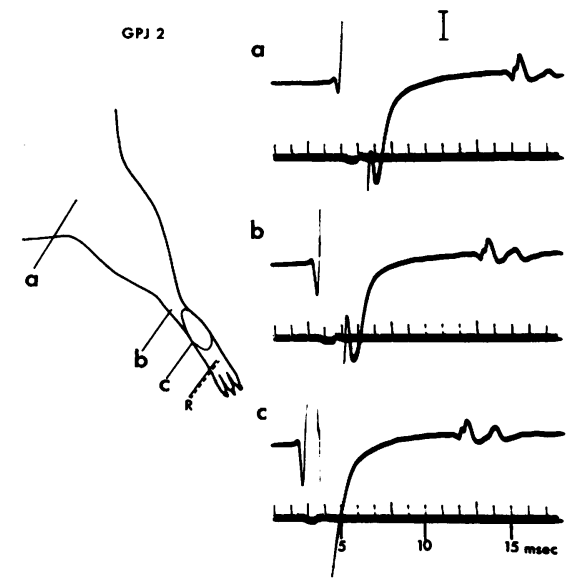

FIG. 4. Early and late responses to nerve stimulation, recorded through a double-core needle electrode in a group $C$ animal. The stimuli, delivered through cathodes $a, b$, and $c$, occurred 1 msec. before the beginning of the sweep in each case. Calibration- $500 \mu \mathrm{V}$.

there was a relatively large early deflection, the latency of which increased from 2.1 msec. with stimulation in the foot to $3.0 \mathrm{msec}$. with stimulation at the ankle and $4.3 \mathrm{msec}$. with stimulation in the thigh. In addition a small late deflection due to a single motor unit was present with a latency which increased from $11.6 \mathrm{msec}$. to $12.8 \mathrm{msec}$. and 14.8 msec. when the stimulus was moved from the foot to the ankle and thigh respectively.

The results of this and three similar experiments are shown in Figure 5 . If the early potentials are regarded as being due to unaffected or relatively mildly affected motor units and the late potentials as being due to severely affected units, then it is clear that in each case the conduction delay was chiefly in the foot. It can be seen that there was a slight difference in conduction time between affected and unaffected motor fibres in the ankle region and in the leg. This slight increase in conduction time 

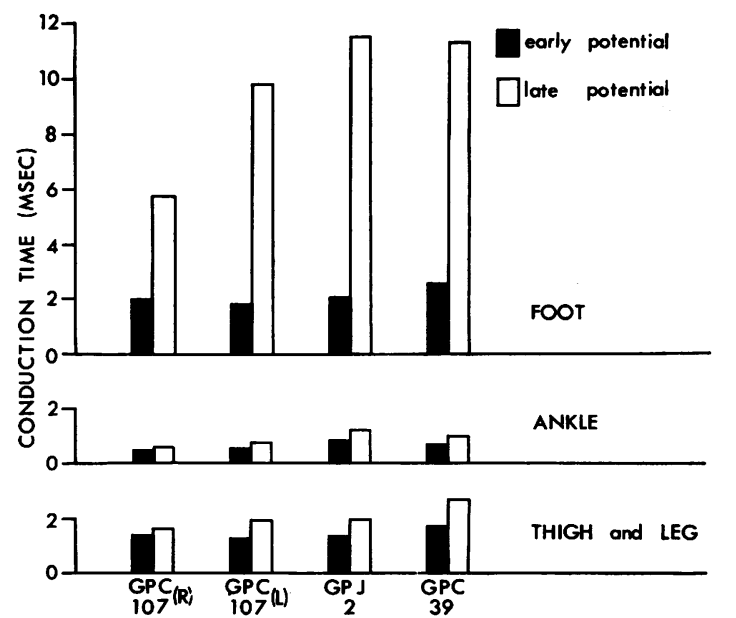

FIG. 5. Conduction times in different segments of the motor nerve for early and late potentials recorded in group $C$ animals. Stimulating and recording arrangements as in Figure 4.

in the proximal parts of affected fibres would not, however, be incompatible with a local lesion in the foot, as changes of this order have been shown to occur proximal to other local lesions, both in man (Ebeling et al., 1960) and in experimental animals (Cragg and Thomas, 1961).

In order to assess the incidence of these abnormalities in different groups of animals, a standard examination procedure was used. In 46 animals records were first made of the muscle responses to maximal stimulation in the thigh and at the ankle; the action potentials were recorded from one of the interosseous muscles through belly-tendon electrodes, attention being paid to the waveform of the response as well as to its latency. The interosseous muscles were then explored with a double-core needle electrode to see whether individual motor units with prolonged latencies were present. When these were found, it was established in each case that the conduction delay was predominantly distal to the ankle.

From Table I it can be seen that individual motor unit potentials with a latency from the ankle of more than $5 \mathrm{msec}$. were relatively rare in animals kept on solid floors, whereas such potentials were common in the group of animals kept on wire-mesh floors. A grossly dispersed muscle response to nerve stimulation was never seen in the former although it was present in about one-third of the animals in the latter group.

In the first animals to be examined, those kept on solid floors were in large pens, whereas the animals on wire-mesh fiooring were in small individual
TABLE I

INCIDENCE OF ABNORMAL FINDINGS IN CAGED (c) AND UNCAGED (A AND B) ANIMALS

\begin{tabular}{lcccc} 
& & & \multicolumn{2}{c}{ Abnormality $^{1}$} \\
\cline { 5 - 6 } Group & $\begin{array}{l}\text { Legs } \\
\text { Examined }\end{array}$ & Normal & Grade I & Grade II \\
\hline A & 24 & 22 & 2 & 0 \\
B & 28 & 21 & 7 & 0 \\
C & 25 & 1 & 16 & 8
\end{tabular}

${ }^{1}$ Abnormalities graded as follows:

I Single motor unit potentials with distal latencies greater than 5 msec. found with double-core needle. Belly-tendon records show no dispersion.

II Single late units found as in I; in addition belly-tendon records show prolonged polyphasic muscle responses.

cages. A later experiment was therefore set up in which 16 animals of similar age and weight were divided into two groups and kept for nine to 10 months in individual cages of similar size but with different flooring, one type of cage having a solid floor covered with sawdust (group D), and the other having a wire-mesh floor similar to that used in previous experiments (group C). The standard examination described above was used and the results are shown in Table II. It can be seen that animals kept on sawdust in small cages fared no worse than those kept in large pens, whereas animals on wire-mesh flooring again gave a high proportion of abnormal findings, the incidence of these abnormalities being similar to that shown in Table I.

\section{TABLE II}

INCIDENCE OF ABNORMAL FINDINGS IN CAGED ANIMALS ON WIRE-MESH FLOORING (C) AND ON SOLID FLOORING (D)

\begin{tabular}{|c|c|c|c|c|}
\hline \multirow[b]{2}{*}{ Group } & \multirow{2}{*}{$\begin{array}{l}\text { Legs } \\
\text { Examined }\end{array}$} & \multirow[b]{2}{*}{ Normal } & \multicolumn{2}{|c|}{ Abnormality 1} \\
\hline & & & Grade I & Grade II \\
\hline $\begin{array}{l}\text { C } \\
\mathbf{D}\end{array}$ & $\begin{array}{l}16 \\
16\end{array}$ & $\begin{array}{r}1 \\
13\end{array}$ & $\begin{array}{l}8 \\
3\end{array}$ & $\begin{array}{l}7 \\
0\end{array}$ \\
\hline
\end{tabular}

${ }^{1}$ Grading of abnormality as in Table I.

\section{HISTOLOGICAL RESULTS}

Transverse sections of the posterior tibial nerve and of the plantar nerves in the foot were examined in four young animals reared and kept on solid floors (group A), and in four animals which had been kept for nine to 12 months on wire-mesh floors (group C). All four animals in the latter group showed dispersion of the muscle response to nerve stimulation. In most animals the posterior tibial and plantar nerves were removed and processed separately, but in some cases they were obtained in continuity so that sections at one or two millimetre intervals could be cut from the mid-calf to the distal part of the foot. 


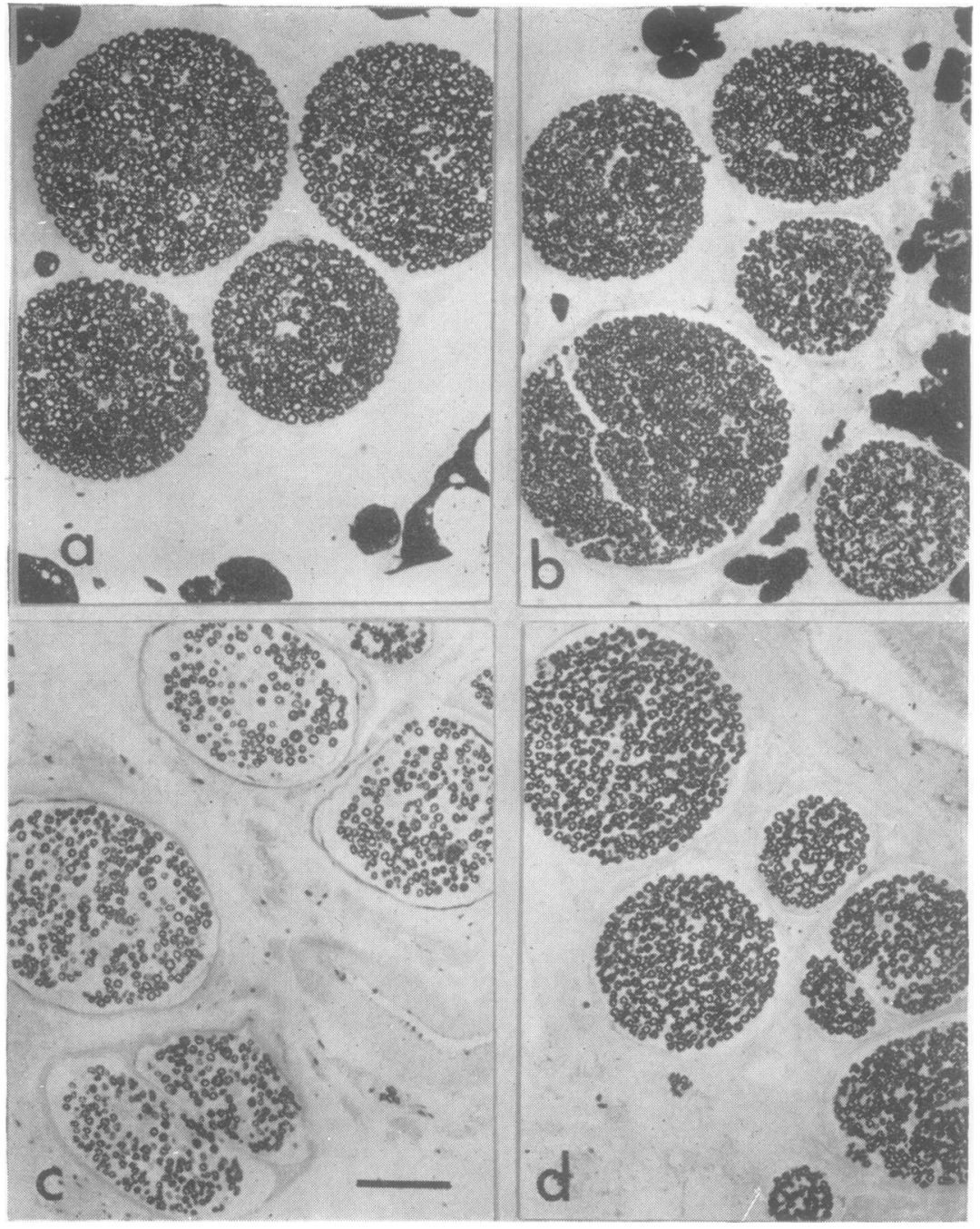

FIG. 6. T.S. of nerves fixed in Flemming's solution and stained by a modified Weigert method to show myelin sheaths. (a) Posterior tibial nerve above ankle and (c) plantar nerve from mid-foot in a group C animal (GPC 120). (b) Posterior tibial nerve above ankle and $(d)$ plantar nerve from midfoot in a group $A$ animal GP 146). Scale-100 $\mu$.

Three out of the four group $C$ animals showed loss of myelinated fibres when compared with group A. This change was maximal in the plantar nerves of the foot but in one animal it extended to the region of the ankle. The posterior tibial nerve above the ankle was unaffected in all cases. The severity of the change in the foot itself varied in different animals. In two of them, loss of myelin was only present in one or two fascicles; in the most severe example, which is shown in Figure 6, there was a diffuse change affecting all the fascicles in the nerve examined. In sections which were counterstained by the van Gieson method, the perineurial sheaths and blood vessels of the affected fascicles appeared normal.
In order to establish whether the loss of myelin in tranverse sections was due to Wallerian degeneration or to segmental demyelination, single fibres were examined in teased preparations. $A b$ normalities were found in nine out of 11 nerves from group $C$ animals; these were not present in nerves from three animals in group $A$.

In most cases affected nerve fibres appeared to become abnormal approximately $1 \mathrm{~cm}$. below the ankle at the level of the tarso-metatarsal joints. The commonest lesion was demyelination, which might remain localized to the paranodal region (Fig. 7) or which might spread to involve one or more complete internodal segments (Fig. 8). In most of the lesions some remyelination had occurred but a few 
examples were seen in which the axon still appeared to be completely denuded of myelin. With osmium staining early remyelination could be recognized not only by the formation of new nodes of Ranvier but also by the appearance of Schmidt-Lantermann incisures, resulting in dark transverse bands across the new myelin sheaths. For example, the fibre shown in Figure 8, which was taken from the middle of the sole, shows a $2.5 \mathrm{~mm}$. length in the process of remyelination with the formation of eight new internodes. This indicates a mean internodal length of approximately $0.3 \mathrm{~mm}$. for the remyelinated segments, which may be contrasted with an internodal length of 0.7 to $0.8 \mathrm{~mm}$. for the intact segments proximal and distal to the affected region.

In addition to fibres which showed evidence of segmental demyelination, others were seen which appeared to be undergoing Wallerian degeneration (Fig. 7a). In a single fibre stained with osmium tetroxide this process could be recognized by the presence of irregularly spaced ovoids of degenerating myelin extending for several millimetres, no normal segments being found distal to the degenerating part. At the upper level of the lesion,

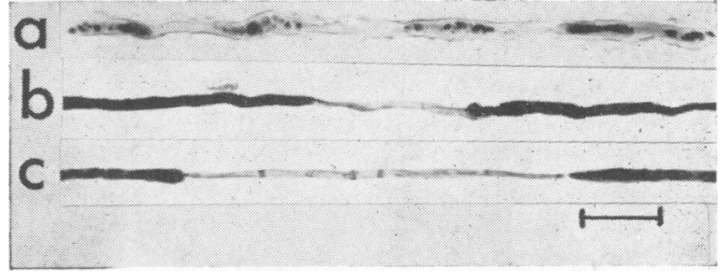

FIG. 7. Single fibres stained with $1 \%$ osmium tetroxide from plantar nerve of the opposite foot to that shown in Figure 6c (GPC 120). (a) Wallerian degeneration, (b) paranodal demyelination, (c) remyelination with formation of a short intercalated segment. Scale $-50 \mu$.

paranodal demyelination was sometimes seen to involve the last few segments above the point at which Wallerian degeneration commenced. This change is similar to that described by Lubińska (1961) in single fibres after experimental nerve crush.

Single fibres which appeared to be regenerating after Wallerian degeneration were also seen in the foot. These showed thin myelin sheaths with closely but regularly spaced nodes of Ranvier. Such fibres

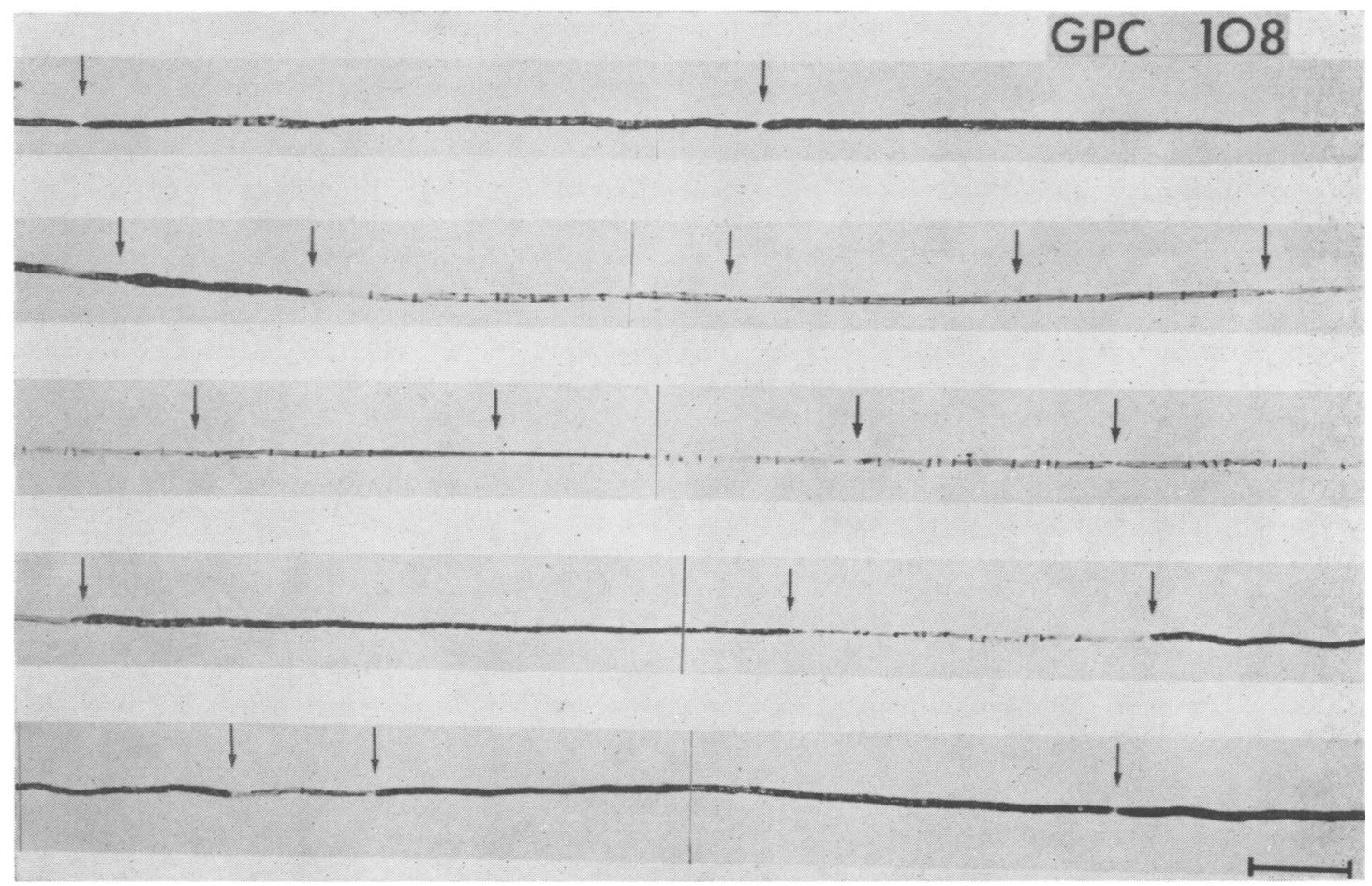

FIG. 8. Consecutive lengths of a single fibre stained with $1 \%$ osmium tetroxide from plantar nerve of a group C animal (GPC 108) showing remyelination with the formation of new short internodes. Each node of Ranvier is marked by an arrow.

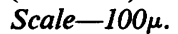


could be distinguished from those undergoing remyelination by the absence of normal internodal segments when they were traced distally.

In mildly affected nerves with only a few abnormal fibres, segmental demyelination alone was usually present, whereas this was mixed with Wallerian degeneration in the more severe lesions.

Longitudinal sections stained by Glees and Marsland's silver technique and counter stained by luxol fast blue proved disappointing in the present study. Few abnormal fibres were seen, and in general the method was less sensitive in detecting mild abnormalities than the examination of single fibres in teased preparations. In the few group $C$ nerves for which it was used, examples of both segmental demyelination and Wallerian degeneration were found.

\section{DISCUSSION}

The relation between the pathological and electrophysiological changes in our animals requires discussion. Segmental demyelination is known to be associated with either reduced conduction velocity or a complete conduction block. This has been shown experimentally in both polyneuropathy (McDonald, 1963; Cragg and Thomas, 1964; Hall, 1964; Morgan-Hughes, 1965, 1966; Fullerton, 1966) and in localized nerve lesions (Mayer and Denny-Brown, 1964; Lehmann and Pretschner, 1966). Although Wallerian degeneration itself is not accompanied by a marked change in conduction velocity (Gutmann and Holubáŕ, 1950; Kaeser and Lambert, 1962), recently regenerated fibres are known to conduct with a greatly reduced velocity which gradually increases as maturation of fibre diameter occurs (Berry, Grundfest, and Hinsey, 1944; Sanders and Whitteridge, 1946). In the present experiments both segmental demyelination and Wallerian degeneration were seen; however, demyelination was the more widespread process and in mildly affected nerves it was sometimes the only pathological change present. This makes it likely that the reduced conduction velocity in the distal parts of the motor fibres was related to demyelination or to early remyelination.

Before accepting that these abnormalities were caused by repeated minor trauma or pressure on the sole of the foot, certain other possibilities must be considered. For example, could there have been any nutritional factor which might have produced a mild generalized neuropathy in these animals, or which might have made the nerves of the foot particularly susceptible to pressure? This seems unlikely since guinea-pigs from several different sources were used for the experiments, and the caged animals were kept in two different animal houses. Furthermore, conduction was normal in the motor fibres to extensor digitorum brevis in animals with severe abnormalities of conduction in the sole. This makes it exceedingly unlikely that any generalized neuropathy was present.

The results shown in Table II make it clear that the size of the cage itself was not a critical factor, the incidence of abnormal findings being related only to the nature of the flooring. It therefore seems reasonable to conclude that repeated pressure on the plantar nerves from the wire-mesh flooring was the cause of both the electrophysiological and the pathological changes which have been described.

This conclusion is relevant to certain human peripheral nerve lesions, in particular to the lesion of the deep branch of the ulnar nerve in the hand, which occurs in patients who suffer mild but repeated trauma to the palm. The trauma need not be severe if it is often repeated. Indeed, one of the patients described by Ebeling et al. (1960) was a secretary who developed a lesion in the palm of the left hand from banging the carriage return lever of her typewriter too vigorously at the end of each line. No pathological studies on these patients were made but slowing of conduction in the distal parts of the motor nerve fibres was a universal finding. This usually affected all the motor fibres to the interosseous muscles, so that the latency of the first deflection of the muscle response was greatly increased. However, in one patient (case 7 of Ebeling et $a l$.) the findings were virtually identical with those described in the present paper, the initial deflection of the muscle response to nerve stimulation having a normal latency but the response itself being greatly dispersed and prolonged due to the presence of late units. It is therefore reasonable to suggest that the underlying pathological changes in the deep branch of the ulnar nerve are likely to be similar to those found in the plantar nerves of our guinea-pigs.

One further point may be made in relation to the present results. The animals kept on solid floors showed occasional late potentials, their incidence being higher in old animals than in young ones. This is relevant to the general question as to whether changes in the peripheral nerves of elderly persons are likely to be due to the ageing process itself or to some related factor such as trauma or vascular degeneration. The localized nature of the abnormality demonstrated in the present series suggests that trauma as well as age itself contributed to the increased incidence of abnormal findings in our old animals.

SUMMARY AND CONCLUSIONS

It has been found that guinea-pigs confined to small 
cages with wire-mesh flooring develop pathological changes in the plantar nerves of the hind foot. These changes, which appear to be due to pressure from the floor of the cage, resulted in slowing of conduction in the distal parts of the motor fibres to the interosseous muscles.

The histological appearances of the plantar nerves in animals with abnormalities of conduction were mainly those of segmental demyelination but in most cases some Wallerian degeneration was also present.

The fact that pressure applied to the sole of the foot can produce local demyelination in the plantar nerves of the guinea-pig may be relevant to man. A rather similar lesion occurs in patients who suffer repeated trauma to the deep branch of the ulnar nerve in the hand, and it may be suggested that this is also likely to involve segmental demyelination.

We wish to thank the National Fund for Research into Poliomyelitis and other Crippling Diseases for a generous grant to one of us (R.W.G.). We also wish to thank Mr. W. Hinkes and Miss C. A. Botwright for technical assistance.

\section{REFERENCES}

Berry, C. M., Grundfest, H., and Hinsey, J. C. (1944). The electrical activity of regenerating nerves in the cat. J. Neurophysiol., 7, 103-115.

Cragg, B. G., and Thomas, P. K. (1961). Changes in conduction velocity and fibre size proximal to peripheral nerve lesions. J. Physiol. (Lond.), 157, 315-327.

- - (1964). Changes in nerve conduction in experimental allergic neuritis. $J$. Neurol. Neurosurg. Psychiat., 27, 106-115.

Culling, C. F. A. (1963). Handbook of Histopathological Techniques, 2nd ed. Butterworths, London.

Ebeling, P., Gilliatt, R. W., and Thomas, P. K. (1960). A clinical and electrical study of ulnar nerve lesions in the hand. J. Neurol. Neurosurg. Psychiat., 23, 1-9.

Fullerton, P. M. (1966). Chronic peripheral neuropathy produced by lead poisoning in guinea-pigs. J. Neuropath. exp. Neurol., 25, 214-236.

_- and Gilliatt, R. W. (1965). Changes in nerve conduction in caged guinea-pigs. J. Physiol. (Lond.), 178, 47-48 $P$.

Gutmann, E., and Holubář, J. (1950). The degeneration of peripheral nerve fibres. J. Neurol. Neurosurg. Psychiat., 13, 89-105.

diameters in the regeneration of peripheral nerves. J. Physiol. (Lond.), 101, 489-518.

Hall, J. I. (1964). Studies on conduction in demyelinated peripheral nerves in the guinea-pig. Ph.D. Thesis, University of Edinburgh.

Kaeser, H. E., and Lambert, E. H. (1962). Nerve function studies in experimental polyneuritis. Electroenceph. clin. Neurophysiol., suppl. 22, 29-35.

Lehmann, H. J., and Pretschner, D. P. (1966). Experimentelle Untersuchungen zum Engpasssyndrom peripherer Nerven. Dtsch. Z. Nervenheilk., 188, 308-330.

Lubinska, L. (1961). Demyelination and remyelination in the proximal parts of regenerating nerve fibers. J. comp. Neurol., 117, 275-289.

McDonald, W. I. (1963). The effects of experimental demyelination on conduction in peripheral nerve: A histological and electrophysiological study. II. Electrophysiological observations. Brain, 86, 501-524.

Mayer, R. F., and Denny-Brown, D. (1964). Conduction velocity in peripheral nerve during experimental demyelination in the cat. Neurology (Minneap.), 14, 714-726.

Morgan-Hughes, J. A. (1965). Changes in motor nerve conduction velocity in diphtheritic polyneuritis. Riv. Pat. nerv. ment., 86, 253-260.

- (1966). Diphtheritic polyneuritis in the guinea-pig. M.D. Thesis, University of Cambridge.

Russell, W. R., and Whitty, C. W. M. (1947). Traumatic neuritis of the deep palmar branch of the ulnar nerve. Lancet, 1, 828-829.

Sanders, F. K., and Whitteridge, D. (1946). Conduction velocity and myelin thickness in regenerating nerve fibres. J. Physiol. (Lond.), $105,152 \cdot 174$.

Short, D. J., and Gammage, L. (1959). New diet for laboratory animals. Brit. med. J., 1, 511-512.

Simpson, J. A. (1956). Electrical signs in the diagnosis of carpal tunnel and related syndromes. J. Neurol. Neurosurg. Psychiat., $19,275-280$.

Thomas, P. K. (1955). Growth changes in the myelin sheath of peripheral nerve fibres in fishes. Proc. roy. Soc. B., 143, 380-391. 